\title{
Boundary value problems for fractional difference equations with three-point fractional sum boundary conditions
}

\section{Thanin Sitthiwirattham ${ }^{1 *}$, Jessada Tariboon ${ }^{1}$ and Sotiris K Ntouyas ${ }^{2}$}

"Correspondence: tst@kmutnb.ac.th 'Department of Mathematics, Faculty of Applied Science, King Mongkut's University of Technology, North Bangkok, Bangkok, Thailand

Full list of author information is

available at the end of the article

\section{Abstract}

In this paper, we consider a discrete fractional boundary value problem of the form

$$
\left\{\begin{array}{l}
\Delta^{\alpha} x(t)=f(t+\alpha-1, x(t+\alpha-1)), \quad t \in[0, T]_{\mathbb{N}_{0}}:=\{0,1, \ldots, T\}, \\
x(\alpha-2)=0, \quad x(\alpha+T)=\Delta^{-\beta} x(\eta+\beta),
\end{array}\right.
$$

where $1<\alpha \leq 2, \beta>0, \eta \in \mathbb{N}_{\alpha-2, \alpha+T-1}:=\{\alpha-2, \alpha-1, \ldots, \alpha+T-2, \alpha+T-1\}$ and $f:[\alpha-1, \ldots, \alpha+T-1]_{\mathbb{N}_{\alpha-1}} \times \mathbb{R} \rightarrow \mathbb{R}$ is a continuous function. Existence and uniqueness of the solutions are proved by using the contraction mapping theorem, the nonlinear contraction theorem and Schaefer's fixed point theorem. Some illustrative examples are also presented.

MSC: $34 \mathrm{~A} 08 ; 26 \mathrm{~A} 33$

Keywords: fractional difference equations; boundary value problem; existence; uniqueness; fixed point theorems

\section{Introduction}

Fractional calculus is an emerging field recently drawing attention from both theoretical and applied disciplines. Fractional order differential equations play a vital role in describing many phenomena related to physics, chemistry, mechanics, control systems, flow in porous media, electrical networks, mathematical biology and viscoelasticity. For a reader interested in the systematic development of the topic, we refer to the books [1-3]. A variety of results on initial and boundary value problems of fractional differential equations and inclusions can easily be found in the literature on the topic. For some recent results, we can refer to [4-12] and references cited therein.

Discrete fractional calculus and fractional difference equations represent a very new area for researchers. Some real-world phenomena are being studied with the help of discrete fractional operators. A good account of papers dealing with discrete fractional boundary value problems can be found in [13-26] and references cited therein.

Goodrich in [22] considered a discrete fractional boundary value problem of the form

$$
\left\{\begin{array}{l}
-\Delta^{v} x(t)=f(t, x(t+v-1)), \quad t \in[0, T]_{\mathbb{N}_{0}}:=\{0,1, \ldots, T\}, \\
x(v-2)=0, \quad x(v+T)=g(x),
\end{array}\right.
$$

@2013 Sitthiwirattham et al;; licensee Springer. This is an Open Access article distributed under the terms of the Creative Commons Attribution License (http://creativecommons.org/licenses/by/2.0), which permits unrestricted use, distribution, and reproduction in any medium, provided the original work is properly cited. 
where $1<v \leq 2, f:[v-2, \ldots, v+T-1]_{\mathbb{N}_{v-2}} \times \mathbb{R} \rightarrow \mathbb{R}$ is a continuous function and $g: C([v-$ $\left.2, v+T]_{\mathbb{N}_{\nu-2}}, \mathbb{R}\right)$ is a given function. Existence and uniqueness of solutions are obtained by the contraction mapping theorem, the Brouwer theorem and the Guo-Krasnoselskii theorem.

Pan et al. in [26] examined the fractional boundary value problem

$$
\left\{\begin{array}{l}
-\Delta_{v-\mu}^{v} x(t)=f(t, x(t+v-1)), \quad t \in\{0,1, \ldots, T+M\} \\
x(v-N)=\cdots=x(v-3)=0 \\
x(v-2)=g(x) \\
\Delta_{v-N}^{\mu} x(T+M+v-\mu)=0
\end{array}\right.
$$

where $v \geq 2, N \in \mathbb{N}$ so that $N-1<v \leq N, 1 \leq \mu<v, M \in \mathbb{N}$ so that $M-1<v \leq M, f$ : $\{0,1, \ldots, T+M\} \times \mathbb{R} \rightarrow \mathbb{R}$ is continuous and nonnegative for $x \geq 0$, and $g: C([\nu-N, \ldots, T+$ $M+v], \mathbb{R})$ is a given function. Existence and uniqueness of solutions are obtained by the contraction mapping theorem and the Brouwer theorem.

In this paper we consider the nonlinear discrete fractional boundary value problem of the form

$$
\left\{\begin{array}{l}
\Delta^{\alpha} x(t)=f(t+\alpha-1, x(t+\alpha-1)), \quad t \in[0, T]_{\mathbb{N}_{0}}:=\{0,1, \ldots, T\}, \\
x(\alpha-2)=0, \quad x(\alpha+T)=\Delta^{-\beta} x(\eta+\beta),
\end{array}\right.
$$

where $1<\alpha \leq 2, \beta>0, \eta \in \mathbb{N}_{\alpha-2, \alpha+T-1}:=\{\alpha-2, \alpha-1, \ldots, \alpha+T-2, \alpha+T-1\}$ and $f$ : $[\alpha-1, \ldots, \alpha+T-1]_{\mathbb{N}_{\alpha-1}} \times \mathbb{R} \rightarrow \mathbb{R}$ is a continuous function.

The plan of this paper is as follows. In Section 2 we recall some definitions and basic lemmas. Also, we derive a representation for the solution to (1.3) by converting the problem to an equivalent summation equation. In Section 3, using this representation, we prove existence and uniqueness of the solutions of boundary value problem (1.3) by the help of the contraction mapping theorem, the nonlinear contraction theorem and Schaefer's fixed point theorem. Some illustrative examples are presented in Section 4.

\section{Preliminaries}

In this section, we introduce notations, definitions and lemmas which are used in the main results.

Definition 2.1 We define the generalized falling function by $t^{\underline{\alpha}}:=\frac{\Gamma(t+1)}{\Gamma(t+1-\alpha)}$, for any $t$ and $\alpha$, for which the right-hand side is defined. If $t+1-\alpha$ is a pole of the gamma function and $t+1$ is not a pole, then $t^{\underline{\alpha}}=0$.

Definition 2.2 The $\alpha$ th fractional sum of a function $f$, for $\alpha>0$, is defined by

$$
\Delta^{-\alpha} f(t)=\Delta^{-\alpha} f(t ; a):=\frac{1}{\Gamma(\alpha)} \sum_{s=a}^{t-\alpha}(t-\sigma(s))^{\frac{\alpha-1}{}} f(s)
$$

for $t \in\{a+\alpha, a+\alpha+1, \ldots\}:=\mathbb{N}_{a+\alpha}$ and $\sigma(s)=s+1$. We also define the $\alpha$ th fractional difference for $\alpha>0$ by $\Delta^{\alpha} f(t):=\Delta^{N} \Delta^{\alpha-N} f(t)$, where $t \in \mathbb{N}_{a+\alpha}$ and $N \in \mathbb{N}$ is chosen so that $0 \leq N-1<\alpha \leq N$. 
Lemma 2.1 Let $t$ and $\alpha$ be any numbers for which $t^{\underline{\alpha}}$ and $t^{\underline{\alpha-1}}$ are defined. Then $\Delta t^{\underline{\alpha}}=$ $\alpha t^{\alpha-1}$.

Lemma 2.2 Let $0 \leq N-1<\alpha \leq N$. Then

$$
\Delta^{-\alpha} \Delta^{\alpha} y(t)=y(t)+C_{1} t^{\underline{\alpha-1}}+C_{2} t^{\underline{\alpha-2}}+\cdots+C_{N} t^{\frac{\alpha-N}{\underline{ }}},
$$

for some $C_{i} \in \mathbb{R}$, with $1 \leq i \leq N$.

To define the solution of boundary value problem (1.3), we need the following lemma which deals with linear variant of boundary value problem (1.3) and gives a representation of the solution.

Lemma 2.3 Let

$$
\sum_{s=1}^{\eta-\alpha+2} \frac{(\eta+\beta-s-\alpha+1) \frac{\beta-1}{\Gamma} \Gamma(s+\alpha-1)}{\Gamma(\beta) \Gamma(s)} \neq \frac{\Gamma(\alpha+T+1)}{\Gamma(T+2)}
$$

and $h:[\alpha-1, \ldots, \alpha+T-1]_{\mathbb{N}_{\alpha-1}} \rightarrow \mathbb{R}$ be given. Then the problem

$$
\left\{\begin{array}{l}
\Delta^{\alpha} x(t)=h(t+\alpha-1), \quad t \in[0, T]_{\mathbb{N}_{0}}, \alpha \in(1,2], \\
x(\alpha-2)=0, \\
x(\alpha+T)=\Delta^{-\beta} x(\eta+\beta), \quad \eta \in \mathbb{N}_{\alpha-2, \alpha+T-1}, \beta>0,
\end{array}\right.
$$

has a unique solution

$$
\begin{aligned}
x(t)= & -\frac{t^{\frac{\alpha-1}{\Lambda}}}{\Lambda(\alpha)}\left[\frac{1}{\Gamma(\beta)} \sum_{s=\alpha}^{\eta} \sum_{\xi=0}^{s-\alpha}(\eta+\beta-\sigma(s))^{\frac{\beta-1}{}}(s-\sigma(\xi))^{\frac{\alpha-1}{h}} h(\xi+\alpha-1)\right. \\
& \left.-\sum_{s=0}^{T}(T+\alpha-\sigma(s))^{\frac{\alpha-1}{h}} h(s+\alpha-1)\right] \\
& +\frac{1}{\Gamma(\alpha)} \sum_{s=0}^{t-\alpha}(t-\sigma(s))^{\frac{\alpha-1}{h}} h(s+\alpha-1)
\end{aligned}
$$

where

$$
\Lambda=\sum_{s=1}^{\eta-\alpha+2} \frac{(\eta+\beta-s-\alpha+1) \frac{\beta-1}{\Gamma} \Gamma(s+\alpha-1)}{\Gamma(\beta) \Gamma(s)}-\frac{\Gamma(\alpha+T+1)}{\Gamma(T+2)} .
$$

Proof From Lemma 2.2, we find that a general solution for (2.2) can be written as

$$
x(t)=C_{1} t^{\frac{\alpha-1}{2}}+C_{2} t^{\frac{\alpha-2}{2}}+\Delta^{-\alpha} h(t+\alpha-1)
$$

for $t \in[\alpha-2, \alpha+T]_{\mathbb{N}_{\alpha-2}}$. Applying the first boundary condition of (2.2) and using ( $\alpha-$ 2) $\frac{\alpha-1}{=} \frac{\Gamma(\alpha-1)}{\Gamma(0)}=0$, we have $C_{2}=0$. So,

$$
x(t)=C_{1} t^{\frac{\alpha-1}{}}+\Delta^{-\alpha} h(t+\alpha-1) .
$$


Using the fractional sum of order $\beta>0$ for (2.6), we obtain

$$
\begin{aligned}
\Delta^{-\beta} x(t)= & \frac{1}{\Gamma(\beta)} \sum_{s=\alpha-2}^{t-\beta}(t-\sigma(s)) \frac{\beta-1}{x}(s)=\frac{C_{1}}{\Gamma(\beta)} \sum_{s=\alpha-2}^{t-\beta}(t-\sigma(s))^{\frac{\beta-1}{2}} s^{\alpha-1} \\
& +\frac{1}{\Gamma(\beta) \Gamma(\alpha)} \sum_{s=\alpha-2}^{t-\beta} \sum_{\xi=0}^{s-\alpha}(t-\sigma(s))^{\frac{\beta-1}{}}(s-\sigma(\xi))^{\frac{\alpha-1}{h}} h(\xi+\alpha-1) .
\end{aligned}
$$

The second condition of (2.2) implies

$$
\begin{aligned}
\Delta^{-\beta} x(\eta+\beta)= & \frac{C_{1}}{\Gamma(\beta)} \sum_{s=\alpha-2}^{\eta}(\eta+\beta-\sigma(s))^{\frac{\beta-1}{}} s^{\alpha-1} \\
& +\frac{1}{\Gamma(\beta) \Gamma(\alpha)} \sum_{s=\alpha-2}^{\eta} \sum_{\xi=0}^{s-\alpha}(\eta+\beta-\sigma(s))^{\frac{\beta-1}{}}(s-\sigma(\xi))^{\frac{\alpha-1}{}} h(\xi+\alpha-1) \\
= & C_{1}(\alpha+T)^{\frac{\alpha-1}{}}+\frac{1}{\Gamma(\alpha)} \sum_{s=0}^{T}(T+\alpha-\sigma(s))^{\frac{\alpha-1}{h}} h(s+\alpha-1) .
\end{aligned}
$$

Solving the above equation for a constant $C_{1}$, we get

$$
\begin{aligned}
C_{1}= & \frac{1}{\Lambda \Gamma(\alpha)} \sum_{s=0}^{T}(T+\alpha-\sigma(s))^{\frac{\alpha-1}{2}} h(s+\alpha-1) \\
& -\frac{1}{\Lambda \Gamma(\beta) \Gamma(\alpha)} \sum_{s=\alpha-2}^{\eta} \sum_{\xi=0}^{s-\alpha}(\eta+\beta-\sigma(s))^{\frac{\beta-1}{}}(s-\sigma(\xi))^{\frac{\alpha-1}{h}} h(\xi+\alpha-1),
\end{aligned}
$$

where $\Lambda$ is defined by (2.4). From the fact that $\sum_{j}^{i}=0$ for $i<j$, we have

$$
\begin{aligned}
C_{1}= & \frac{1}{\Lambda \Gamma(\alpha)} \sum_{s=0}^{T}(T+\alpha-\sigma(s))^{\frac{\alpha-1}{}} h(s+\alpha-1) \\
& -\frac{1}{\Lambda \Gamma(\beta) \Gamma(\alpha)} \sum_{s=\alpha}^{\eta} \sum_{\xi=0}^{s-\alpha}(\eta+\beta-\sigma(s))^{\frac{\beta-1}{}}(s-\sigma(\xi))^{\frac{\alpha-1}{}} h(\xi+\alpha-1) .
\end{aligned}
$$

Substituting a constant $C_{1}$ into (2.6), we obtain (2.3).

\section{Main results}

In this section, we wish to establish the existence results for problem (1.3). To accomplish this, we define $C\left([\alpha-2, \alpha+T]_{\mathbb{N}_{\alpha-2}}, \mathbb{R}\right)$, the Banach space of all function $x$ with the norm defined by $\|x\|=\max \left\{|x(t)|, t \in[\alpha-2, \alpha+T]_{\mathbb{N}_{\alpha-2}}\right\}$ and also define an operator $F: C([\alpha-$ $\left.2, \alpha+T]_{\mathbb{N}_{\alpha-2}}, \mathbb{R}\right) \rightarrow C\left([\alpha-2, \alpha+T]_{\mathbb{N}_{\alpha-2}}, \mathbb{R}\right)$ by

$$
\begin{aligned}
F x(t)= & -\frac{t^{\frac{\alpha-1}{\Lambda}}}{\Lambda \Gamma(\alpha)}\left[\frac{1}{\Gamma(\beta)} \sum_{s=\alpha}^{\eta} \sum_{\xi=0}^{s-\alpha}(\eta+\beta-\sigma(s))^{\frac{\beta-1}{}}\right. \\
& \times(s-\sigma(\xi))^{\frac{\alpha-1}{}} f(\xi+\alpha-1, x(\xi+\alpha-1))
\end{aligned}
$$




$$
\begin{aligned}
& \left.-\sum_{s=0}^{T}(T+\alpha-\sigma(s))^{\frac{\alpha-1}{}} f(s+\alpha-1, x(s+\alpha-1))\right] \\
& +\frac{1}{\Gamma(\alpha)} \sum_{s=0}^{t-\alpha}(t-\sigma(s))^{\frac{\alpha-1}{}} f(s+\alpha-1, x(s+\alpha-1)),
\end{aligned}
$$

for $t \in[\alpha-2, \alpha+T]_{\mathbb{N}_{\alpha-2}}$, where $\Lambda \neq 0$ is defined by (2.4). It is easy to see that problem (1.3) has solutions if and only if the operator $F$ has fixed points.

Theorem 3.1 Suppose that there exists a constant $\gamma>0$ such that $|f(t, x)-f(t, y)| \leq \gamma|x-y|$ for each $t \in[\alpha-2, \alpha+T]_{\mathbb{N}_{\alpha-2}}$ and $x, y \in \mathbb{R}$. If

$$
\gamma \Omega<1
$$

where

$$
\begin{aligned}
\Omega= & \frac{\Gamma(T+\alpha+1)}{|\Lambda| \Gamma(\alpha) \Gamma(T+2)}\left[\frac{1}{\Gamma(\beta)} \sum_{s=\alpha}^{\eta} \sum_{\xi=0}^{s-\alpha}(\eta+\beta-\sigma(s))^{\frac{\beta-1}{}}(s-\sigma(\xi))^{\frac{\alpha-1}{}}\right. \\
& \left.+\sum_{s=0}^{T}(T+\alpha-\sigma(s))^{\frac{\alpha-1}{}}\right]+\frac{\Gamma(T+\alpha+1)}{\Gamma(T+1) \Gamma(\alpha+1)},
\end{aligned}
$$

then problem (1.3) has a unique solution in $[\alpha-2, \alpha+T]_{\mathbb{N}_{\alpha-2}}$.

Proof Firstly, we transform problem (1.3) into a fixed point problem, $x=F x$, where the operator $F: C\left([\alpha-2, \alpha+T]_{\mathbb{N}_{\alpha-2}}, \mathbb{R}\right) \rightarrow C\left([\alpha-2, \alpha+T]_{\mathbb{N}_{\alpha-2}}, \mathbb{R}\right)$ is defined by (3.1). Then, for any $x, y \in C\left([\alpha-2, \alpha+T]_{\mathbb{N}_{\alpha-2}}, \mathbb{R}\right)$, we have

$$
\begin{aligned}
& |(F x)(t)-(F y)(t)| \\
& \leq \frac{\gamma\|x-y\|}{|\Lambda| \Gamma(\alpha)}\left[\frac{1}{\Gamma(\beta)} \sum_{s=\alpha}^{\eta} \sum_{\xi=0}^{s-\alpha}(\eta+\beta-\sigma(s))^{\frac{\beta-1}{}}(s-\sigma(\xi))^{\frac{\alpha-1}{2}}\right. \\
& \left.+\sum_{s=0}^{T}(T+\alpha-\sigma(s))^{\frac{\alpha-1}{}}\right] \max _{t \in[\alpha-2, \alpha+T]_{\mathbb{N}_{\alpha-2}}} t \frac{\alpha-1}{t} \\
& +\frac{\gamma\|x-y\|}{\Gamma(\alpha)} \max _{t \in[\alpha-2, \alpha+T]_{\mathbb{N}_{\alpha-2}}} \sum_{s=0}^{t-\alpha}(t-\sigma(s))^{\frac{\alpha-1}{}} \\
& \leq \frac{\gamma\|x-y\|}{|\Lambda| \Gamma(\alpha)}\left[\frac{1}{\Gamma(\beta)} \sum_{s=\alpha}^{\eta} \sum_{\xi=0}^{s-\alpha}(\eta+\beta-\sigma(s))^{\frac{\beta-1}{}}(s-\sigma(\xi))^{\frac{\alpha-1}{}}\right. \\
& +\sum_{s=0}^{T}(T+\alpha-\sigma(s))^{\left.\frac{\alpha-1}{}\right]} \max _{t \in[\alpha-2, \alpha+T]_{\mathbb{N}_{\alpha-2}}} \frac{\Gamma(t+1)}{\Gamma(t-\alpha+2)} \\
& +\frac{\gamma\|x-y\|}{\Gamma(\alpha)} \max _{t \in[\alpha-2, \alpha+T]_{\mathbb{N}_{\alpha-2}}}\left[\frac{-1}{\alpha}(t-s)^{\underline{\alpha}}\right]_{s=0}^{t-\alpha+1} \\
& \leq \frac{\gamma\|x-y\|}{|\Lambda| \Gamma(\alpha)}\left[\frac{1}{\Gamma(\beta)} \sum_{s=\alpha}^{\eta} \sum_{\xi=0}^{s-\alpha}(\eta+\beta-\sigma(s))^{\frac{\beta-1}{}}(s-\sigma(\xi))^{\underline{\alpha-1}}\right.
\end{aligned}
$$




$$
\begin{aligned}
& \left.+\sum_{s=0}^{T}(T+\alpha-\sigma(s))^{\alpha-1}\right] \frac{\Gamma(T+\alpha+1)}{\Gamma(T+2)} \\
& +\gamma\|x-y\| \max _{t \in[\alpha-2, \alpha+T]_{\mathbb{N}_{\alpha-2}}} \frac{\Gamma(t+1)}{\Gamma(t-\alpha+1) \Gamma(\alpha+1)} \\
& \leq \frac{\gamma\|x-y\| \Gamma(T+\alpha+1)}{|\Lambda| \Gamma(\alpha) \Gamma(T+2)}\left[\frac{1}{\Gamma(\beta)} \sum_{s=\alpha}^{\eta} \sum_{\xi=0}^{s-\alpha}(\eta+\beta-\sigma(s))^{\frac{\beta-1}{(}(s-\sigma(\xi))^{\frac{\alpha-1}{}}}\right. \\
& \left.+\sum_{s=0}^{T}(T+\alpha-\sigma(s))^{\underline{\alpha-1}}\right]+\gamma\|x-y\| \frac{\Gamma(T+\alpha+1)}{\Gamma(T+1) \Gamma(\alpha+1)} \\
& =\gamma \Omega\|x-y\| .
\end{aligned}
$$

Thus

$$
\|F x-F y\| \leq \gamma \Omega\|x-y\| .
$$

Therefore, $F$ is a contraction. Hence, by the Banach fixed point theorem, we get that $F$ has a fixed point which is a unique solution of problem (1.3) on $t \in[\alpha-2, \alpha+T]_{\mathbb{N}_{\alpha-2}}$.

Next, we can still deduce the existence of a solution to (1.3). We shall use nonlinear contraction to accomplish this.

Definition 3.1 Let $E$ be a Banach space and let $F: E \rightarrow E$ be a mapping. $F$ is said to be a nonlinear contraction if there exists a continuous nondecreasing function $\Psi: R^{+} \rightarrow R^{+}$ such that $\Psi(0)=0$ and $\Psi(\rho)<\rho$ for all $\rho>0$ with the property

$$
\|F x-F y\| \leq \Psi(\|x-y\|), \quad \forall x, y \in E
$$

Lemma 3.1 (Boyd and Wong [27]) Let E be a Banach space and let $F: E \rightarrow E$ be a nonlinear contraction. Then $F$ has a unique fixed point in $E$.

Theorem 3.2 Suppose that there exists a continuous function $h:[\alpha-2, \alpha+T]_{\mathbb{N}_{\alpha-2}} \rightarrow \mathbb{R}^{+}$ such that $|f(t, x)-f(t, y)| \leq h(t) \frac{|x-y|}{H^{*}+|x-y|}$ for all $t \in[\alpha-2, \alpha+T]_{\mathbb{N}_{\alpha-2}}$ and $x, y \geq 0$, where

$$
\begin{aligned}
H^{*}= & \frac{\Gamma(T+\alpha+1)}{|\Lambda| \Gamma(\alpha) \Gamma(T+2)}\left[\frac{1}{\Gamma(\beta)} \sum_{s=\alpha}^{\eta} \sum_{\xi=0}^{s-\alpha}(\eta+\beta-\sigma(s))^{\frac{\beta-1}{1}}\right. \\
& \left.\times(s-\sigma(\xi))^{\frac{\alpha-1}{h}} h(\xi+\alpha-1)+\sum_{s=0}^{T}(T+\alpha-\sigma(s))^{\frac{\alpha-1}{2}} h(s+\alpha-1)\right] \\
& +\frac{1}{\Gamma(\alpha)} \sum_{s=0}^{T}(T+\alpha-\sigma(s))^{\frac{\alpha-1}{h}} h(s+\alpha-1)
\end{aligned}
$$

and $\Lambda$ is defined in (2.4).

Then boundary value problem (1.3) has a unique solution. 
Proof Let the operator $F: C\left([\alpha-2, \alpha+T]_{\mathbb{N}_{\alpha-2}}, \mathbb{R}\right) \rightarrow C\left([\alpha-2, \alpha+T]_{\mathbb{N}_{\alpha-2}}, \mathbb{R}\right)$ be defined by (3.1). We define a continuous nondecreasing function $\Psi: \mathbb{R}^{+} \rightarrow \mathbb{R}^{+}$by

$$
\Psi(\rho)=\frac{H^{*} \rho}{H^{*}+\rho}, \quad \forall \rho \geq 0,
$$

such that $\Psi(0)=0$ and $\Psi(\rho)<\rho$ for all $\rho>0$.

Let $x, y \in C\left([\alpha-2, \alpha+T]_{\mathbb{N}_{\alpha-2}}\right)$. Then we get

$$
|f(s, x(s))-f(s, y(s))| \leq \frac{h(s)}{H^{*}} \Psi(\|x-y\|),
$$

so that

$$
\begin{aligned}
\mid F x(t) & -F y(t) \mid \\
\leq & \frac{t^{\alpha-1}}{|\Lambda| \Gamma(\alpha)}\left[\frac{1}{\Gamma(\beta)} \sum_{s=\alpha}^{\eta} \sum_{\xi=0}^{s-\alpha}(\eta+\beta-\sigma(s))^{\frac{\beta-1}{}}\right. \\
& \times(s-\sigma(\xi))^{\underline{\alpha-1}} h(\xi+\alpha-1) \frac{|x(\xi+\alpha-1)-y(\xi+\alpha-1)|}{H^{*}+|x(\xi+\alpha-1)-y(\xi+\alpha-1)|} \\
& \left.+\sum_{s=0}^{T}(T+\alpha-\sigma(s))^{\frac{\alpha-1}{h}} h(s+\alpha-1) \times \frac{|x(s+\alpha-1)-y(s+\alpha-1)|}{H^{*}+|x(s+\alpha-1)-y(s+\alpha-1)|}\right] \\
& +\frac{1}{\Gamma(\alpha)} \sum_{s=0}^{t-\alpha}(t-\sigma(s))^{\frac{\alpha-1}{h}} h(s+\alpha-1) \frac{|x(s+\alpha-1)-y(s+\alpha-1)|}{H^{*}+|x(s+\alpha-1)-y(s+\alpha-1)|} \\
\leq & \left\{\frac { \Gamma ( T + \alpha + 1 ) } { | \Lambda | \Gamma ( \alpha ) \Gamma ( T + 2 ) } \left[\frac{1}{\Gamma(\beta)} \sum_{s=\alpha}^{\eta} \sum_{\xi=0}^{s-\alpha}(\eta+\beta-\sigma(s))^{\frac{\beta-1}{2}}\right.\right. \\
& \left.\times(s-\sigma(\xi))^{\frac{\alpha-1}{2}} h(\xi+\alpha-1)+\sum_{s=0}^{T}(T+\alpha-\sigma(s))^{\frac{\alpha-1}{2}} h(s+\alpha-1)\right] \\
& \left.+\frac{1}{\Gamma(\alpha)} \sum_{s=0}^{T}(T+\alpha-\sigma(s))^{\frac{\alpha-1}{h} h(s+\alpha-1)}\right\} \frac{\|x-y\|}{H^{*}+\|x-y\|}
\end{aligned}
$$

for $t \in[\alpha-2, \alpha+T]_{\mathbb{N}_{\alpha-2}}$. From (3.4), it follows that $\|F x-F y\| \leq \Psi(\|x-y\|)$. Hence $F$ is a nonlinear contraction. Therefore, by Lemma 3.1, the operator $F$ has a unique fixed point in $C\left([\alpha-2, \alpha+T]_{\mathbb{N}_{\alpha-2}}\right)$, which is a unique solution of problem (1.3).

The following result is based on Schaefer's fixed point theorem.

Theorem 3.3 Suppose that there exists a constant $M>0$ such that $|f(t, x)| \leq M$ for each $t \in[\alpha-2, \alpha+T]_{\mathbb{N}_{\alpha-2}}$ and all $x \in \mathbb{R}$.

Then problem (1.3) has at least one solution on $[\alpha-2, \alpha+T]_{\mathbb{N}_{\alpha-2}}$.

Proof We shall use Schaefer's fixed point theorem to prove that the operator $F$ defined by (3.1) has a fixed point. We divide the proof into four steps. 
Step I. Continuity of $F$. Let $x_{n}$ be a sequence such that $x_{n} \rightarrow x$ in $C\left([\alpha-2, \alpha+T]_{\mathbb{N}_{\alpha-2}}\right)$. Then, for each $t \in[\alpha-2, \alpha+T]_{\mathbb{N}_{\alpha-2}}$, we get

$$
\begin{aligned}
& \left|F\left(x_{n}\right)(t)-F(x)(t)\right| \\
& \leq \frac{t^{\alpha-1}}{|\Lambda| \Gamma(\alpha)}\left[\frac{1}{\Gamma(\beta)} \sum_{s=\alpha}^{\eta} \sum_{\xi=0}^{s-\alpha}(\eta+\beta-\sigma(s))^{\beta-1}\right. \\
& \times(s-\sigma(\xi))^{\frac{\alpha-1}{}}\left|f\left(\xi+\alpha-1, x_{n}(\xi+\alpha-1)\right)-f(\xi+\alpha-1, x(\xi+\alpha-1))\right| \\
& +\sum_{s=0}^{T}(T+\alpha-\sigma(s))^{\underline{\alpha-1}} \mid f\left(s+\alpha-1, x_{n}(s+\alpha-1)\right) \\
& -f(s+\alpha-1, x(s+\alpha-1)) \mid] \\
& +\frac{1}{\Gamma(\alpha)} \sum_{s=0}^{t-\alpha}(t-\sigma(s))^{\frac{\alpha-1}{}}\left|f\left(s+\alpha-1, x_{n}(s+\alpha-1)\right)-f(s+\alpha-1, x(s+\alpha-1))\right| \\
& \leq \frac{t^{\frac{\alpha-1}{}}}{|\Lambda| \Gamma(\alpha)}\left[\frac{1}{\Gamma(\beta)} \sum_{s=\alpha}^{\eta} \sum_{\xi=0}^{s-\alpha}(\eta+\beta-\sigma(s))^{\frac{\beta-1}{}}(s-\sigma(\xi))^{\frac{\alpha-1}{2}}\right. \\
& \times \max _{\xi \in[\alpha-2, \alpha+T]_{\mathbb{N}_{\alpha-2}}}\left|f\left(\xi+\alpha-1, x_{n}(\xi+\alpha-1)\right)-f(\xi+\alpha-1, x(\xi+\alpha-1))\right| \\
& +\sum_{s=0}^{T}(T+\alpha-\sigma(s))^{\alpha-1} \max _{s \in[\alpha-2, \alpha+T]_{\mathbb{N}_{\alpha-2}}} \mid f\left(s+\alpha-1, x_{n}(s+\alpha-1)\right) \\
& -f(s+\alpha-1, x(s+\alpha-1)) \mid] \\
& +\frac{1}{\Gamma(\alpha)} \sum_{s=0}^{t-\alpha}(t-\sigma(s))^{\frac{\alpha-1}{}} \max _{s \in[\alpha-2, \alpha+T]_{\mathbb{N}_{\alpha-2}}} \mid f\left(s+\alpha-1, x_{n}(s+\alpha-1)\right) \\
& -f(s+\alpha-1, x(s+\alpha-1)) \mid \text {. }
\end{aligned}
$$

Since $f$ is a continuous function, we have $\left\|F x_{n}-F x\right\| \rightarrow 0$ as $n \rightarrow 0$. This means that $F$ is continuous.

Step II. F maps bounded sets into bounded sets in $C\left([\alpha-2, \alpha+T]_{\mathbb{N}_{\alpha-2}}\right)$. Let us prove that for any $R>0$, there exists a positive constant $L$ such that for each $x \in B_{R}=\{x \in C([\alpha-$ $\left.\left.2, \alpha+T]_{\mathbb{N}_{\alpha-2}}, \mathbb{R}\right):\|x\| \leq R\right\}$, we have $\|F x\| \leq L$. Indeed, for any $x \in B_{R}$, we obtain

$$
\begin{aligned}
|F x(t)| \leq & \frac{t^{\underline{\alpha-1}}}{|\Lambda| \Gamma(\alpha)}\left[\frac{1}{\Gamma(\beta)} \sum_{s=\alpha}^{\eta} \sum_{\xi=0}^{s-\alpha}(\eta+\beta-\sigma(s))^{\frac{\beta-1}{}}\right. \\
& \times(s-\sigma(\xi))^{\frac{\alpha-1}{}}|f(\xi+\alpha-1, x(\xi+\alpha-1))| \\
& \left.+\sum_{s=0}^{T}(T+\alpha-\sigma(s))^{\frac{\alpha-1}{}}|f(s+\alpha-1, x(s+\alpha-1))|\right] \\
& +\frac{1}{\Gamma(\alpha)} \sum_{s=0}^{t-\alpha}(t-\sigma(s))^{\underline{\alpha-1}}|f(s+\alpha-1, x(s+\alpha-1))| .
\end{aligned}
$$


Therefore,

$$
\begin{aligned}
|F x(t)| \leq & \frac{M t^{\alpha-1}}{|\Lambda| \Gamma(\alpha)}\left[\frac{1}{\Gamma(\beta)} \sum_{s=\alpha}^{\eta} \sum_{\xi=0}^{s-\alpha}(\eta+\beta-\sigma(s))^{\frac{\beta-1}{}}(s-\sigma(\xi))^{\frac{\alpha-1}{}}\right. \\
& \left.+\sum_{s=0}^{T}(T+\alpha-\sigma(s))^{\frac{\alpha-1}{}}\right]+\frac{M}{\Gamma(\alpha)} \sum_{s=0}^{t-\alpha}(t-\sigma(s))^{\frac{\alpha-1}{}} .
\end{aligned}
$$

Hence, we deduce that

$$
\begin{aligned}
\|F x\| \leq & \frac{M \Gamma(T+\alpha+1)}{|\Lambda| \Gamma(\alpha) \Gamma(T+2)}\left[\frac{1}{\Gamma(\beta)} \sum_{s=\alpha}^{\eta} \sum_{\xi=0}^{s-\alpha}(\eta+\beta-\sigma(s))^{\frac{\beta-1}{}}(s-\sigma(\xi))^{\frac{\alpha-1}{}}\right. \\
& \left.+\sum_{s=0}^{T}(T+\alpha-\sigma(s))^{\frac{\alpha-1}{}}\right]+M \frac{\Gamma(T+\alpha+1)}{\Gamma(T+1) \Gamma(\alpha+1)} \\
\leq & M \Omega:=L,
\end{aligned}
$$

where $\Omega$ is defined by (3.3).

Step III. $F\left(B_{R}\right)$ is equicontinuous with $B_{R}$ defined as in Step II. For any $\varepsilon>0$, there exist $t_{1}, t_{2} \in[\alpha-2, \alpha+T]_{\mathbb{N}_{\alpha-2}}, t_{1} \leq t_{2}$ such that

$$
\begin{aligned}
& \frac{\left|t_{2}^{\alpha-1}-t_{1}^{\alpha-1}\right| M}{|\Lambda| \Gamma(\alpha)}\left[\frac{1}{\Gamma(\beta)} \sum_{s=\alpha}^{\eta} \sum_{\xi=0}^{s-\alpha}(\eta+\beta-\sigma(s))^{\frac{\beta-1}{}}(s-\sigma(\xi))^{\frac{\alpha-1}{}}\right. \\
& \left.\quad+\sum_{s=0}^{T}(T+\alpha-\sigma(s))^{\frac{\alpha-1}{}}\right]+\frac{M}{\Gamma(\alpha)}\left[\frac{\Gamma\left(t_{2}+1\right)}{\Gamma\left(t_{2}-\alpha+1\right)}-\frac{\Gamma\left(t_{1}+1\right)}{\Gamma\left(t_{1}-\alpha+1\right)}\right]<\varepsilon .
\end{aligned}
$$

Then we have

$$
\begin{aligned}
& \left|F x\left(t_{2}\right)-F x\left(t_{1}\right)\right| \\
& =\mid-\frac{t_{2}^{\alpha-1}-t_{1}^{\alpha-1}}{\Lambda \Gamma(\alpha)}\left[\frac{1}{\Gamma(\beta)} \sum_{s=\alpha}^{\eta} \sum_{\xi=0}^{s-\alpha}(\eta+\beta-\sigma(s))^{\frac{\beta-1}{}}\right. \\
& \times(s-\sigma(\xi))^{\frac{\alpha-1}{}} f(\xi+\alpha-1, x(\xi+\alpha-1)) \\
& \left.-\sum_{s=0}^{T}(T+\alpha-\sigma(s))^{\frac{\alpha-1}{}} f(s+\alpha-1, x(s+\alpha-1))\right] \\
& +\frac{1}{\Gamma(\alpha)}\left[\sum_{s=0}^{t_{1}-\alpha}\left(\left(t_{2}-\sigma(s)\right)^{\frac{\alpha-1}{}}-\left(t_{1}-\sigma(s)\right)^{\frac{\alpha-1}{}}\right) f(s+\alpha-1, x(s+\alpha-1))\right. \\
& \left.+\sum_{s=t_{1}-\alpha+1}^{t_{2}-\alpha}\left(t_{2}-\sigma(s)\right)^{\frac{\alpha-1}{}} f(s+\alpha-1, x(s+\alpha-1))\right] \mid \\
& \leq \frac{\left|t_{2}^{\alpha-1}-t_{1}^{\alpha-1}\right| M}{|\Lambda| \Gamma(\alpha)}\left[\frac{1}{\Gamma(\beta)} \sum_{s=\alpha}^{\eta} \sum_{\xi=0}^{s-\alpha}(\eta+\beta-\sigma(s))^{\frac{\beta-1}{}}(s-\sigma(\xi))^{\frac{\alpha-1}{}}\right. \\
& \left.+\sum_{s=0}^{T}(T+\alpha-\sigma(s))^{\frac{\alpha-1}{}}\right]+\frac{M}{\Gamma(\alpha)}\left[\sum _ { s = 0 } ^ { t _ { 1 } - \alpha } \left(\left(t_{2}-\sigma(s)\right)^{\frac{\alpha-1}{}}-\left(t_{1}-\sigma(s)\right)^{\left.\frac{\alpha-1}{}\right)}\right.\right.
\end{aligned}
$$




$$
\begin{aligned}
& \left.+\sum_{s=t_{1}-\alpha+1}^{t_{2}-\alpha}\left(t_{2}-\sigma(s)\right)^{\frac{\alpha-1}{1}}\right] \\
= & \frac{\left|t_{2}^{\alpha-1}-t_{1}^{\alpha-1}\right| M}{|\Lambda| \Gamma(\alpha)}\left[\frac{1}{\Gamma(\beta)} \sum_{s=\alpha}^{\eta} \sum_{\xi=0}^{s-\alpha}(\eta+\beta-\sigma(s))^{\frac{\beta-1}{}}(s-\sigma(\xi))^{\frac{\alpha-1}{}}\right. \\
& \left.+\sum_{s=0}^{T}(T+\alpha-\sigma(s))^{\frac{\alpha-1}{}}\right]+\frac{M}{\Gamma(\alpha)}\left[\frac{\Gamma\left(t_{2}+1\right)}{\Gamma\left(t_{2}-\alpha+1\right)}-\frac{\Gamma\left(t_{1}+1\right)}{\Gamma\left(t_{1}-\alpha+1\right)}\right] \\
\leq & \varepsilon .
\end{aligned}
$$

This means that the set $F\left(B_{R}\right)$ is an equicontinuous set. As a consequence of Steps I to III together with the Arzelá-Ascoli theorem, we get that $F: C\left([\alpha-2, \alpha+T]_{\mathbb{N}_{\alpha-2}}\right) \rightarrow C([\alpha-$ $2, \alpha+T]_{\mathbb{N}_{\alpha-2}}$ ) is completely continuous.

Step IV. A priori bounds. We show that the set

$$
E=\left\{x \in C\left([\alpha-2, \alpha+T]_{\mathbb{N}_{\alpha-2}}, \mathbb{R}\right): x=\lambda F x \text { for some } 0<\lambda<1\right\}
$$

is bounded.

Let $x \in E$. Then $x(t)=\lambda(F x)(t)$ for some $0<\lambda<1$. Thus, for each $t \in[\alpha-2, \alpha+T]_{\mathbb{N}_{\alpha-2}}$, we have

$$
\begin{aligned}
\lambda(F x)(t)= & -\frac{\lambda t^{\frac{\alpha-1}{\Lambda}}}{\Lambda \Gamma(\alpha)}\left[\frac{1}{\Gamma(\beta)} \sum_{s=\alpha}^{\eta} \sum_{\xi=0}^{s-\alpha}(\eta+\beta-\sigma(s))^{\frac{\beta-1}{}}\right. \\
& \times(s-\sigma(\xi))^{\frac{\alpha-1}{}} f(\xi+\alpha-1, x(\xi+\alpha-1)) \\
& \left.-\sum_{s=0}^{T}(T+\alpha-\sigma(s))^{\frac{\alpha-1}{}} f(s+\alpha-1, x(s+\alpha-1))\right] \\
& +\frac{\lambda}{\Gamma(\alpha)} \sum_{s=0}^{t-\alpha}(t-\sigma(s))^{\frac{\alpha-1}{f}} f(s+\alpha-1, x(s+\alpha-1)) .
\end{aligned}
$$

Therefore, for $t \in[\alpha-2, \alpha+T]_{\mathbb{N}_{\alpha-2}}$, we get

$$
\begin{aligned}
& |\lambda(F x)(t)| \leq \frac{\lambda M t^{\underline{\alpha-1}}}{|\Lambda| \Gamma(\alpha)}\left[\frac{1}{\Gamma(\beta)} \sum_{s=\alpha}^{\eta} \sum_{\xi=0}^{s-\alpha}(\eta+\beta-\sigma(s))^{\frac{\beta-1}{(}(s-\sigma(\xi))^{\frac{\alpha-1}{}}}\right. \\
& \left.+\sum_{s=0}^{T}(T+\alpha-\sigma(s))^{\underline{\alpha-1}}\right]+\frac{\lambda M}{\Gamma(\alpha)} \sum_{s=0}^{t-\alpha}(t-\sigma(s))^{\frac{\alpha-1}{2}} \\
& \leq \frac{M \Gamma(T+\alpha+1)}{|\Lambda| \Gamma(\alpha) \Gamma(T+2)}\left[\frac{1}{\Gamma(\beta)} \sum_{s=\alpha}^{\eta} \sum_{\xi=0}^{s-\alpha}(\eta+\beta-\sigma(s))^{\frac{\beta-1}{}}\right. \\
& \times(s-\sigma(\xi))^{\frac{\alpha-1}{2}}+\sum_{s=0}^{T}(T+\alpha-\sigma(s))^{\left.\frac{\alpha-1}{}\right]} \\
& +\frac{M \Gamma(T+\alpha+1)}{\Gamma(T+1) \Gamma(\alpha+1)} \\
& =M \Omega:=L \text {. }
\end{aligned}
$$


This shows that the set $E$ is bounded. As a consequence of Schaefer's fixed point theorem, we conclude that $F$ has a fixed point which is a solution of problem (1.3).

\section{Some examples}

In this section, in order to illustrate our result, we consider some examples.

Example 4.1 Consider the following three-point fractional sum boundary value problem:

$$
\begin{aligned}
& \Delta^{\frac{3}{2}} x(t)=\frac{e^{-\sin ^{2}\left(t+\frac{1}{2}\right)}}{\left(t+\frac{7}{2}\right)^{2}} \cdot \frac{|x|}{|x|+1}, \quad t \in N_{\frac{1}{2}, \frac{9}{2}} \\
& x\left(-\frac{1}{2}\right)=0, \\
& x\left(\frac{11}{2}\right)=\Delta^{-\frac{4}{3}} x\left(\frac{23}{6}\right) .
\end{aligned}
$$

Here $\alpha=\frac{3}{2}, \beta=\frac{4}{3}, \eta=\frac{5}{2}, T=4, f(t, x)=\frac{e^{-\sin ^{2} t}}{(t+3)^{2}} \cdot \frac{|x|}{|x|+1}$. Also, we find

$$
\Lambda=\sum_{s=1}^{3} \frac{\left(\frac{10}{3}-s\right)^{\frac{1}{3}} \Gamma\left(s+\frac{1}{2}\right)}{\frac{1}{3} \Gamma\left(\frac{1}{3}\right) \Gamma(s)}-\frac{\Gamma\left(\frac{13}{2}\right)}{\Gamma(6)}=-283 \neq 0
$$

and

$$
\begin{aligned}
\Omega= & \frac{\Gamma\left(\frac{13}{2}\right)}{|-283| \Gamma\left(\frac{3}{2}\right) \Gamma(6)}\left[\frac{1}{\Gamma\left(\frac{4}{3}\right)} \sum_{s=\frac{3}{2}}^{\frac{5}{2}} \sum_{\xi=0}^{s-\frac{3}{2}}\left(\frac{5}{2}+\frac{4}{3}-\sigma(s)\right)^{\frac{4}{3}-1}(s-\sigma(\xi))^{\frac{3}{2}-1}\right. \\
& \left.+\sum_{s=0}^{4}\left(T+\frac{3}{2}-\sigma(s)\right)^{\frac{3}{2}-1}\right]+\frac{\Gamma\left(\frac{13}{2}\right)}{\Gamma\left(\frac{5}{2}\right) \Gamma(5)} \\
\approx & 10.29 .
\end{aligned}
$$

From $|f(t, x)-f(t, y)| \leq \frac{4}{49}|x-y|$, we have $\gamma=\frac{4}{49}$. We can show that

$$
\gamma \Omega=\frac{4}{49} \times 10.29=0.84<1
$$

Hence, by Theorem 3.1, boundary value problem (4.1)-(4.3) has a unique solution.

Example 4.2 Consider the following three-point fractional sum boundary value problem:

$$
\begin{aligned}
& \Delta^{\frac{3}{2}} x(t)=\frac{1}{10}\left(t+\frac{1}{2}\right) e^{-\frac{1}{100}\left(t+\frac{1}{2}\right)\left|x\left(t+\frac{1}{2}\right)\right|}, \quad t \in N_{\frac{1}{2}, \frac{9}{2}} \\
& x\left(-\frac{1}{2}\right)=0, \\
& x\left(\frac{11}{2}\right)=\Delta^{-\frac{4}{3}} x\left(\frac{23}{6}\right) .
\end{aligned}
$$


Here $\alpha=\frac{3}{2}, \beta=\frac{4}{3}, \eta=\frac{5}{2}, T=4, f(t, x)=\frac{1}{10}(t) e^{-\frac{1}{100} t|x(t)|}, \Lambda=-283 \neq 0, \Omega=1.91$. It is clear that $X|f(t, x)| \leq \frac{11}{20}<1=M$ for $t \in N_{\frac{1}{2}}, \frac{9}{2}$. Thus, we conclude from Theorem 3.3 that (4.4)(4.6) has at least one solution.

\section{Competing interests}

The authors declare that they have no competing interests.

\section{Authors' contributions}

All authors contributed equally in this article. They read and approved the final manuscript.

\section{Author details}

1 Department of Mathematics, Faculty of Applied Science, King Mongkut's University of Technology, North Bangkok, Bangkok, Thailand. ²Department of Mathematics, University of loannina, loannina, 451 10, Greece.

\section{Authors' information}

The third author is a member of Nonlinear Analysis and Applied Mathematics (NAAM)-Research Group at King Abdulaziz University, Jeddah, Saudi Arabia.

\section{Acknowledgements}

The present paper was done while J Tariboon and T Sitthiwirattham visited the Department of Mathematics of the University of loannina, Greece. It is a pleasure for them to thank Professor SK Ntouyas for his warm hospitality. This research of J Tariboon and T Sitthiwirattham is supported by King Mongkut's University of Technology North Bangkok, Thailand.

Received: 8 May 2013 Accepted: 17 September 2013 Published: 07 Nov 2013

\section{References}

1. Podlubny, I: Fractional Differential Equations. Academic Press, San Diego (1999)

2. Kilbas, AA, Srivastava, HM, Trujillo, JJ: Theory and Applications of Fractional Differential Equations. North-Holland Mathematics Studies, vol. 204. Elsevier, Amsterdam (2006)

3. Baleanu, D, Diethelm, K, Scalas, E, Trujillo, JJ: Fractional Calculus Models and Numerical Methods. Series on Complexity, Nonlinearity and Chaos. World Scientific, Boston (2012)

4. Guezane-Lakoud, A, Khaldi, R: Solvability of a three-point fractional nonlinear boundary value problem. Differ. Equ. Dyn. Syst. 20, 395-403 (2012)

5. Guezane-Lakoud, A, Khaldi, R: Positive solution to a higher order fractional boundary value problem with fractional integral condition. Rom. J. Math. Comput. Sci. 2,41-54 (2012)

6. Kaufmann, E: Existence and nonexistence of positive solutions for a nonlinear fractional boundary value problem Discrete Contin. Dyn. Syst. 2009, suppl.,416-423 (2009)

7. Wang, J, Xiang, H, Liu, Z: Positive solution to nonzero boundary values problem for a coupled system of nonlinear fractional differential equations. Int. J. Differ. Equ. 2010, Article ID 186928 (2010)

8. Bai, Z: On positive solutions of a nonlocal fractional boundary value problem. Nonlinear Anal. 72, 916-924 (2010)

9. Sudsutad, W, Tariboon, J: Boundary value problems for fractional differential equations with three-point fractional integral boundary conditions. Adv. Differ. Equ. 2012, 93 (2012)

10. Ntouyas, SK: Boundary value problems for nonlinear fractional differential equations and inclusions with nonlocal and fractional integral boundary conditions. Opusc. Math. 33, 117-138 (2013)

11. Guezane-Lakoud, A, Khaldi, R: Solvability of a fractional boundary value problem with fractional integral condition. Nonlinear Anal. 75, 2692-2700 (2012)

12. Ahmad, B, Ntouyas, SK, Assolani, A: Caputo type fractional differential equations with nonlocal Riemann-Liouville integral boundary conditions. J. Appl. Math. Comput. 41, 339-350 (2013)

13. Baleanu, D, Mustafa, OG, O'Regan, D: On a fractional differential equation with infinitely many solutions. Adv. Differ. Equ. 2012, 145 (2012)

14. Liu, Y, Ahmad, B, Agarwal, RP: Existence of solutions for a coupled system of nonlinear fractional differential equations with fractional boundary conditions on the half line. Adv. Differ. Equ. 2013, 46 (2013)

15. Ahmad, B, Ntouyas, SK, Alsaedi, A: A study of nonlinear fractional differential equations of arbitrary order with Riemann-Liouville type multi-strip boundary conditions. Math. Probl. Eng. 2013, Article ID 320415 (2013)

16. Zhang, L, Ahmad, B, Wang, G, Agarwal, RP: Nonlinear fractional integro-differential equations on unbounded domains in a Banach space. J. Comput. Appl. Math. 249, 51-56 (2013)

17. Ahmadian, A, Suleiman, M, Salahshour, S, Baleanu, D: A Jacobi operational matrix for solving a fuzzy linear fractional differential equation. Adv. Differ. Equ. 2013, 104 (2013)

18. Ferreira, R: Existence and uniqueness of solution to some discrete fractional boundary value problems of order less than one. J. Differ. Equ. Appl. 19, 712-718 (2013)

19. Ahmad, B, Nieto, JJ: Basic theory of nonlinear third-order q-difference equations and inclusions. Math. Model. Anal. $18,122-135$ (2013)

20. Pongarm, N, Asawasamrit, S, Tariboon, J: Sequential derivatives of nonlinear q-difference equations with three-point q-integral boundary conditions. J. Appl. Math. 2013, Article ID 605169 (2013)

21. Atici, F, Eloe, P: Two-point boundary value problems for finite fractional difference equations. J. Differ. Equ. Appl. 17 445-456 (2011) 
22. Goodrich, CS: Existence and uniqueness of solutions to a fractional difference equation with nonlocal conditions. Comput. Math. Appl. 61, 191-202 (2011)

23. Goodrich, CS: Existence of a positive solution to a system of discrete fractional boundary value problems. Appl. Math. Comput. 217, 4740-4753 (2011)

24. Goodrich, CS: On a discrete fractional three-point boundary value problem. J. Differ. Equ. Appl. 18, 397-415 (2012)

25. Abdeljawad, T: On Riemann and Caputo fractional differences. Comput. Math. Appl. 62, 1602-1611 (2011)

26. Pan, Y, Han, Z, Sun, S, Huang, Z: The existence and uniqueness of solutions to boundary value problems of fractional difference equations. Math. Sci. 6, 7 (2012)

27. Boyd, DW, Wong, JSW: On nonlinear contractions. Proc. Am. Math. Soc. 20, 458-464 (1969)

10.1186/1687-1847-2013-296

Cite this article as: Sitthiwirattham et al.: Boundary value problems for fractional difference equations with three-point fractional sum boundary conditions. Advances in Difference Equations 2013, 2013:296

\section{Submit your manuscript to a SpringerOpen ${ }^{\circ}$ journal and benefit from:}

- Convenient online submission

- Rigorous peer review

- Immediate publication on acceptance

- Open access: articles freely available online

- High visibility within the field

- Retaining the copyright to your article 